\title{
EL MERCADO TURISTICO DE ACAPULCO: ESTUDIO DEL PERFIL DE LOS TURISTAS
}

\author{
Miguel Guerrero Ocampo \\ Agustín Santiago Moreno \\ Carlos Bouza Herrera \\ Universidad Autónoma de Guerrero, Acapulco. México \\ Universidad de LA Habana, La Habana, Cuba. \\ https://doi.org/10.17979/redma.2009.01.02.4687
}

\section{RESUMEN}

Se realiza un estudio sobre el perfil de los turistas que acuden a Acapulco. Este estudio se basa en una muestra de 399 turistas. Los resultados obtenidos permiten identificar el perfil del turismo en términos de su satisfacción de las oferta del mercado.

\section{Palabras Clave:}

Mercado turístico, categorías de satisfacción, análisis de datos.

\begin{abstract}
ACAPULCO'S TOURISM MARKET: TOURISTS PROFILE ANALYSIS

The profile of the tourists visiting Acapulco is made. This study uses a sample of 399 tourists. The obtained results allow the identification of the satisfaction profile of the tourism in terms of the offers of the market
\end{abstract} Keywords:

Tourism market, satisfaction categories, data analysis 


\section{Introducción}

Muchos estudios de mercado se basan en investigaciones que buscan razones o conocimientos para poder manejarle, sin detenerse a detallar si las políticas a implementar están limitadas en términos de la realidad, dada la esencia misma del fenómeno. El mercado turístico se ha buscado explicar de diversas maneras al contabilizar el porcentaje de turistas que regresan satisfechos a su lugar de origen, pero sin detenerse a explicar de una manera más puntual las consecuencias personales de tal satisfacción, lo cual está asociado con el impacto que la satisfacción tiene en su campo de la experiencia. Sin ese conocimiento cualquier política de mercado deja de escudriñar metodológicamente el campo de la experiencia (realidad experiencial inmediata) del turista al momento que se encontraba en el Puerto de Acapulco, viviendo una realidad distinta a la que vive todos los días. Es nuestro interés explorar y describir aquellos elementos que han ido conformando esa experiencia, tanto por el hecho de ser un turista como por encontrarse en determinados lugares turísticos de Acapulco. En este trabajo se supone que lo más importante para el turista al encontrarse en un determinado lugar de Acapulco es alcanzar su autorrealización.

Los supuestos sobre los que elaboramos nuestras tesis parten de que cada turista posee una experiencia acerca de sí mismo y de los acontecimientos de su vida y que integra toda su realidad psíquica en su campo de la experiencia. Esta es conformada a través de sus percepciones, sentimientos-emociones y fantasías. Por ello consideráremos lo que ocurre en el campo de la experiencia de los turistas que visitan Acapulco para determinar el grado en el que las realidades psíquicas son integradas a partir de la interpretación o reinterpretación que ellos hacen de las experiencias vividas en ciertos lugares de Acapulco, acontecida en siete lugares de Acapulco que son la oferta del mercado considerada más atrayente. Además, se trata de dar una descripción 
de la estructura de esa experiencia, lograda a través del desdoblamiento que el visitante hace en cada uno de los acontecimientos vividos, buscando con ello su autorrealización. Esto permitiría hacer ofertas que estimulen al individuo a partir de sus preferencias. De esta manera y conjugando toda una gama de argumentos teóricos a la hora de conformar los necesarios cambios en la imagen de este destino turístico ante las ofertas de otros polos emergentes como Cancún, entre otros.

El postulado teórico, que sustenta la utilidad de esta investigación para el mercadeo es que para cualquier persona, lo más importante es la experiencia que tiene de un determinado lugar y momento, más que la reacción que pudiera tener del mundo que le rodea. En consecuencia, debido al énfasis que se hace acerca del campo de la experiencia como parte fundamental de la realidad psíquica, el trabajo está enfocado hacia un estudio fenomenológico del turista. Por esta razón, la investigación representa una oportunidad para enunciar de manera diferente lo que es el tiempo libre.

Para hacer el estudio se diseñó un cuestionario que fue aplicado a una muestra seleccionada bajo un muestreo parcialmente probabilístico. Los datos permitieron hacer un estudio de la autorrealización y el auto concepto de los turistas que acuden Acapulco. Esto se hizo para determinar las influencias de los diversos indicadores y de la cuantificación de la fortaleza que estos tenían en la conformación de la imagen de la oferta. Esto permitirá establecer orientaciones sobre los fines de los estudios de mercado que propendan a mejorar la imagen de la oferta de este destino turísticos para mejorar la calidad de los indicadores más importantes. En la sección 2 se dan algunos elementos sobre el comportamiento del turismo en el Puerto de Acapulco en los últimos años. En la sección 3 se establecen algunos enfoques psicológicos que ayudarán a comprender el diseño de la investigación y la importancia de la autorrealización y el autoconcepto para comprender el turismo desde el punto de vista de la psicología del turista como individuo. En la sección 4 se categoriza el 
tiempo libre para poder diseñar su estudio psico-social. Posteriormente se pasa a caracterizar el mercado turístico de Acapulco de acuerdo a las ofertas que brinda al turismo. La sección 6 presenta los principios utiizados para el diseño de la encuesta: cuestionario y modelo de muestreo. El análisis de la $\mathrm{s}$ configuraciones es llevada acabo en la sección 7 donde se establecen las regularidades observadas en los indicadores y se cuantifica su fuerza en la valoración de la autorrealización y en la formación de autoconceptos. Estos resultados dan pie a identificar los indicadores más importantes al discriminar la formación de criterios que deben crear una fidelidad a al polo turístico de Acapulco. Técnicas de Análisis Multivariado fueron utilizadas con este fin.

\section{El mercado turístico de Acapulco}

La afluencia turística en Acapulco presenta una tendencia irregular con algunos repuntes. Así, durante el período 2000 al 2004, el número de visitantes fue de: 4'775,544; 4'596,239; 4'086,114; 3'905,749 y 5'334,110, respectivamente. Ahora, analizando al turismo internacional en general, Teresa de J. Rivas P. (1997:6-84) ofrece datos que nos llevan a observar una tendencia hacia la baja. Por ejemplo, del año 1975 al 1981 la afluencia de este tipo de turismo se mantuvo arriba de los 600 mil, pero en el año 1995 esa cifra había disminuido al $50 \%$. Más recientemente, la D1. Introducción

En este trabajo se presentan los resultados de una investigación llevada a cabo en Acapulco para establecer la valoración de la satisfacción de los turistas.

La afluencia turística en Acapulco presenta una tendencia irregular con algunos repuntes. Así, durante el período 2000 al 2004, el número de visitantes fue de: 4'775,544; 4'596,239; 4'086,114; 3'905,749 y 5'334,110, respectivamente. Ahora, analizando al turismo internacional en general, Teresa de J. Rivas P. (1997:6-84) ofrece datos que nos llevan a observar una tendencia hacia la baja. Por ejemplo, del año 1975 al 1981 la afluencia de este tipo de turismo se 
mantuvo arriba de los 600 mil, pero en el año 1995 esa cifra había disminuido al 50\%. Más recientemente, la Dirección General de Turismo Municipal de Acapulco, reportó que durante el período 2001-2004, el turismo internacional mantuvo esa misma tendencia: 149,035; 106,555; 91,375 y 106114, respectivamente, haciendo un total de 453,079. Dicha tendencia también se ve reflejada en la derrama económica, la cual, en el lustro 2000-2004 las cifras fueron: 12,879' (millones de pesos), 13,817', 13,858', 11,421' y 13,590'; eso mismo se observa con los empleos directos e indirectos. Sin duda que, para que esto ocurra, se han mencionado una variedad de razones, V.gr., la contaminación de la bahía, costos de peaje, tanto de la Autopista del Sol, como del atracadero en el muelle, campañas de desprestigio contra Acapulco, infraestructura insuficiente e inadecuada, etc. Sin embargo, hasta el momento ninguno de estos argumentos se ha ponderado más que el otro, por lo que no se tiene una claridad acerca del aspecto nodal que pudiera atenderse de manera prioritaria a fin de reposicionar al puerto entre los mejores segmentos de mercado.

A partir de la toma de la muestra de 399 turistas se hizo un estudio de las variables autorrealización, autoconcepto y temporalidad, dadas como importantes desde el punto de vista psico-social para el turismo, ver Guerrero (2008). Del estudio se derivan una serie de recomendaciones dirigidas a los prestadores del servicio turístico.

\section{Variables y sucesos.}

Las investigaciones llevadas a cabo sobre el ocio y el tiempo libre, permite ver claramente cuál es el sentido que se le ha ido dando a lo largo de varios años, a uno y otro tiempo y, llama la atención que la mayoría de los teóricos, independientemente de su ideología, concuerda en aspectos fundamentales. 
Más todavía, varios de esos trabajos están sustentados en el humanismo, donde la libertad para, no libertad de, ocupa el lugar central

En este trabajo se estudian las variables autorrealización, el autoconcepto y la temporalidad.

Para ello se definen sub variables que permiten diseccionar la formación de criterios sobre la satisfacción de las expectativas de los turistas en el Puerto de Acapulco como sigue: .

\section{I.- Autorrealización}

1.- Dimensión biológica (el cuerpo)

Saludable-enfermo, Fuerte-débil

2.- Autodeterminación

Descanso-fatiga, Diversión-aburrimiento, Desarrollo de la personalidad-dificultad para el perfeccionamiento; Las hace libremente-es movido por los demás

\section{3.- Autorrealización propiamente}

Su vida está llena de significados-resulta ser un vacío; sus sentimientos los acepta-se asusta de ellos.

4.- Satisfacción de las necesidades

Status social (rango)-pasa desapercibido; Lo aceptan y lo reconocen-le rehúyen; Han sido satisfechas-están insatisfechas; sensación agradable o placentera-de fastidio; El momento y el lugar los disfruta-le son aburridos y, sus fantasías se han cumplido-no se han realizado.

\section{Il.- Autoconcepto}

1.- Percepción de sí mismo

Sí, yo me veo aquí como un turista-No, así no me veo

2.- Lo que cree que los demás ven de él

Creo que los demás me ven como turista-No, creo que así no me ven

\section{III.- Horizonte temporal}

\section{1.- Pasado-futuro}

REDMARKA UIMA-Universidad de A Coruña - CIECID 
Su atención se centra en el pasado recordando cosas-en el futuro haciendo planes

\section{2.- Experiencia del tiempo transcurrido}

Pasó rápidamente-pasó lentamente

El cuestionario permitió obtener información para obtener una valoración de los criterios de los turistas.

Se tomó una muestra en lugares característicos de Acapulco que denominaremos sucesos.

\section{Perfil de los turistas encuestados}

Es conveniente hacer un análisis de las características generales de los turistas investigados con el fin de buscar si existe o no alguna conexión entre las configuraciones de las sub-variables estudiadas y ellas. Tales características se refieren a las siguientes variables:

Variable 1.- Edad.- Estudiar esta variable, básicamente tiene su resonancia en la publicidad que se hará. También es un marco de referencia para las conclusiones acerca del horizonte temporal del mercado representado por los turistas investigados.

Los turistas de este estudio se hallan en un rango de edad que va, de los 18 a los 70 años, siendo la edad promedio general de 30 años. La distribución de frecuencias es dada en la tabla 1.

Respecto a los rangos de edad que aparecen en la tabla 1, tales rangos tienen como base el criterio de los estándares del ciclo productivo de las personas de la clase media mexicana. ${ }^{1}$ Anteriormente ya se hizo el comentario de que el factor económico es un determinante, aparte del tiempo libre, para ser turista.

\footnotetext{
${ }^{1}$ De acuerdo a los estándares del ciclo productivo de una persona, algunas aseguradoras, entre ellas MetLIfe (Agosto 2007), consideran lo siguiente: en el período comprendido entre 19 a 25 años se REDMARKA UIMA-Universidad de A Coruña - CIECID Año 1, Número 2, (2009), pp. 77-110 http://www.redmarka.org/ ISSN 1852-2300 
Tabla 1. Distribución de la edad de los entrevistados

\begin{tabular}{|l|l|l|}
\hline Intervalo de edad & Característica laboral & Por ciento \\
\hline 18-25 años & Dependencia e independencia económica & $35 \%$ \\
\hline 26-35 años & Independencia económica & $44 \%$ \\
\hline 36-55 años & Plenitud económica & $17 \%$ \\
\hline 56-o más años... & Preparación para el retiro laboral o retirado & $3 \%$ \\
\hline
\end{tabular}

De acuerdo a los porcentajes de la tabla 1, el 79\% de los turistas investigados está comprendido entre los 18 y 35 años de edad, predominando los que representan independencia económica. Por tanto toda acción de mercadeo debe tomar en cuenta en su promoción captar la preferencia de los menores de 40 años.

Variable 2.- Estado civil.- El $45.4 \%$ eran solteros y el $41 \%$ casados, haciendo un total del $86.4 \%$. Esta variable sugiere que debe hacerse por igual ofertas para parejas que para personas sin ella.

Variable 3.- Forma de compañía. El 55.4\% de turistas se hallaba acompañado por familiares y amigos, mientras que el $27 \%$ por su pareja y, el $7.5 \%$ solo. Esto sugiere la importancia de hacer ofertas que sean atrayentes para grupos.

Variable 4.- Lugar de origen. El 40.4\% provenía del Distrito Federal, el 10\% del Estado de Morelos, el 7\% del Estado de México e igual porcentaje del Estado de Puebla, el 5\% de Oaxaca y el 4.5\% de Guadalajara, haciendo un total del $74 \%$. Esto hace evidente que el grueso de los turistas son del pais.

encuentran las personas, tanto dependientes como independientes económicamente; de los 26 a los 35 años, están las personas con independencia económica; entre los 36 a los 55 años, se encuentran las personas con plenitud económica y, de los 56 a 65 años es el período de preparación para el retiro laboral.

REDMARKA UIMA-Universidad de A Coruña - CIECID 
Variable 5.- Tipo de ocupación. El 27.3\% son profesionistas, 26\% eran estudiantes, el 18\% empleados, $11 \%$ comerciantes, $4.3 \%$ empresarios y el $1.3 \%$ operadores del volante, dando un total del 88\%. Esto evidencia que el nicho de mercado que acude a Acapulco es fundamentalmente de clase media.

La tabla 2 da cuenta de cinco características generales del turismo a partir de la encuesta.

Tabla 2 Características generales de los entrevistados

\begin{tabular}{|c|c|c|c|c|}
\hline Suc & Edad (\%) & $\begin{array}{l}\text { Estado civil } \\
(\%)\end{array}$ & $\begin{array}{l}\text { Tipo de compañía } \\
(\%)\end{array}$ & ación (\%) \\
\hline $\begin{array}{l}\text { Discoteca } \\
\text { Total }=45 \\
\text { Sexo: } \\
M=20 \\
F=25\end{array}$ & $\begin{array}{l}\text { Promedio } \\
=27.8 \\
(18-25 \square= \\
44 \% \\
26-35=40 \\
36-55=14 \\
56-65 \ldots=2)\end{array}$ & $\begin{array}{l}\text { Soltero =65 } \\
\text { Casado =30 } \\
\text { Otro caso = } \\
5\end{array}$ & $\begin{array}{l}\text { Familiares } \quad y / 0 \\
\text { amistades= } 80 \\
\text { Con su pareja = } 6 \\
\text { Otro caso=14 }\end{array}$ & $\begin{array}{l}\text { Profesionista = } \\
38 \\
\text { Estudiante = } \\
38 \% \\
\text { Comerciante = } \\
9 \\
\text { Empleado = } 7 \\
\text { Otras }=8\end{array}$ \\
\hline $\begin{array}{l}\text { Playa } \\
\text { Total }=157 \\
\text { Sexo: } \\
M=80 \\
F=77\end{array}$ & $\begin{array}{l}\text { Promedio } \\
=30.5 \\
(18-25=14 \\
26-35=52 \\
36-55=30 \\
56- \\
\text { más })=3)\end{array}$ & $\begin{array}{l}\text { Soltero }=43 \\
\text { Casado }=42 \\
\text { Otro caso } \\
=15\end{array}$ & $\begin{array}{l}\text { Familiares } \quad y / 0 \\
\text { amistades } \\
=56 \\
\text { Con su pareja = } 27 \\
\text { Otro caso=17 }\end{array}$ & $\begin{array}{l}\text { Profesionista = } \\
25 \\
\text { Estudiante = } \\
28 \\
\text { Comerciante = } \\
10 \\
\text { Empleado = } 15 \\
\text { Otra }=22\end{array}$ \\
\hline $\begin{array}{l}\frac{\text { Hotel }}{\text { Total }}=65 \\
\text { Sexo: }\end{array}$ & $\begin{array}{l}\text { Promedio } \\
=30.3 \\
(18-25=28\end{array}$ & $\begin{array}{l}\text { Soltero = } 50 \\
\text { Casado = } \\
38\end{array}$ & $\begin{array}{l}\text { Familiares } \\
\text { amistades } \\
=47\end{array}$ & $\begin{array}{l}\text { Profesionista = } \\
30 \\
\text { Estudiante = }\end{array}$ \\
\hline
\end{tabular}




\begin{tabular}{|c|c|c|c|c|}
\hline $\begin{array}{l}M=35 \\
F=30\end{array}$ & $\begin{array}{l}26-35=51 \\
36-55=20 \\
56-65 \ldots=1)\end{array}$ & $\begin{array}{l}\text { Otro } \\
\text { caso }=12\end{array}$ & $\begin{array}{l}\text { Con su pareja = } 41 \\
\text { Otro caso=12 }\end{array}$ & $\begin{array}{l}22 \\
\text { Comerciante }= \\
6 \\
\text { Empleado = } 27 \\
\text { Otra }=11\end{array}$ \\
\hline $\begin{array}{l}\text { CICl } \\
\text { Total }=35^{2} \\
\text { Sexo: } \\
M=21 \\
F=14\end{array}$ & $\begin{array}{l}\text { Promedio } \\
=32 \\
18-25=23 \\
26-35=54 \\
36-55=17 \\
56-65 \ldots=6\end{array}$ & $\begin{array}{l}\text { Soltero = } 37 \\
\text { Casado = } \\
49 \\
\text { Otro } \\
\text { caso }=14\end{array}$ & $\begin{array}{l}\text { Familiares } \\
\text { amistades } \\
=57 \\
\text { Con su pareja = } 20 \\
\text { Otro caso=23 }\end{array}$ & $\begin{array}{l}\text { Profesionista = } \\
21 \\
\text { Estudiante = } \\
24 \\
\text { Comerciante = } \\
15 \\
\text { Empleado = } 15 \\
\text { Otra }=25\end{array}$ \\
\hline $\begin{array}{l}\text { Quebrada } \\
\text { Total = 58 } \\
\text { Sexo: } \\
M=38 \\
F=25\end{array}$ & $\begin{array}{l}\text { Promedio } \\
=33.3 \\
(18-25=36 \\
26-35=2 \oint \\
36-55=31 \\
56-0 \\
\text { más...=7) }\end{array}$ & $\begin{array}{l}\text { Soltero = } 33 \\
\text { Casado = } \\
48 \\
\text { Otro } \\
\text { caso=19 }\end{array}$ & $\begin{array}{l}\text { Familiares } \quad y / 0 \\
\text { amistades } \\
=57 \\
\text { Con su pareja = } 31 \\
\text { Otro caso=12 }\end{array}$ & $\begin{array}{l}\text { Profesionista = } \\
14 \\
\text { Estudiante = } \\
21 \\
\text { Comerciante = } \\
21 \\
\text { Empleado = } 30 \\
\text { Otra }=14\end{array}$ \\
\hline $\begin{array}{l}\text { Bungi } \\
\text { Total = } 11 \\
\text { Sexo: } \\
M=6 \\
F=5\end{array}$ & $\begin{array}{l}\text { Promedio = } \\
24.3 \\
(18-25 E 88 \\
26-35=18 \\
36-55=0 \\
56-\quad 0 \quad \text { más } \\
\ldots=0)\end{array}$ & $\begin{array}{l}\text { Soltero }=91 \\
\text { Casado }=0 \\
\text { Otro caso }=9\end{array}$ & $\begin{array}{l}\text { Familiares } \quad y / 0 \\
\text { amistades } \\
=45 \\
\text { Con su pareja = } 45 \\
\text { Otro caso=10 }\end{array}$ & $\begin{array}{l}\text { Profesionista = } \\
36 \\
\text { Estudiante = } \\
64 \\
\text { Comerciante } \\
=0 \\
\text { Empleado =0 }\end{array}$ \\
\hline
\end{tabular}

${ }^{2}$ Este total de turistas entrevistados tuvo que ver con el hecho de que en el CICI se encontraba un buen número de acapulqueños, lo cual ocasionó un problema metodológico, ya que el azar, en varias ocasiones, no fue la mejor opción.

REDMARKA UIMA-Universidad de A Coruña - CIECID 


\begin{tabular}{|l|l|l|l|l|}
\hline Banana & Promedio & Soltero $=43$ & Familiares y/o & Profesionista = \\
Total = 28 & $=29.8$ & Casado $=$ & amistades & 27 \\
Sexo: & $(18-25=21$ & 54 & $=64$ & Estudiante = \\
$\mathrm{M}=13$ & $26-35 \equiv 68$ & Otro caso=2 & Con su pareja = 29 & 18 \\
$\mathrm{~F}=15$ & $36-55=11$ & & Otro caso=7 & Comerciante = \\
& $56-$ o más $=$ & & & 13 \\
& $0)$ & & & Empleado = 32 \\
& & & & \\
& & & & \\
\hline
\end{tabular}

En cuanto al lugar de procedencia, los resultados son los siguientes:

Discos .- Del D.F. 40\% y 31\% de los estados de Morelos, Oaxaca y Puebla. Ellos dan un total $71 \%$. .

Playa.- El 36\% provenía del DF y el 31\% de los Estados de: Morelos, Edo. de México, Oaxaca y Puebla, haciendo un total del $67 \%$.

Hotel.- Provenía del D.F. el 52\%; del Estado de Morelos el 13\% y el 19\% de los Estados de México, Oaxaca y Puebla. Total 84\%.

CICl.- El 20\% provenía de D.F; el 17\% del Estado de México; 11\% por igual, de los Estado de Guerrero y Puebla y, el 9\% de Morelos, e igual porcentaje para el Estado de Oaxaca, haciendo un total del $78 \%$.

Quebrada.- Del D.F. provenía el 41\% y 10\% de Puebla. Los Estados de Morelos y Guanajuato, cada uno de ellos con el $7 \%$, haciendo un total del 58\%

Bungi.- En relación al lugar de origen, el 64\% procedía del D.F.; el resto correspondió a los Estados de Michoacán, Puebla y Guerrero. 
Banana.- El 54\% provino del D.F., el 14\% de Guadalajara, 7\% de Michoacán y también $7 \%$ de Puebla, representando un total de 82\%.

Esto establece de que regiones acuden con más frecuencia y diferenciar la oferta en la publicidad.

Por otra parte del análisis etario se obtiene que no hay duda de que el Bungi es para los jóvenes, pues el $82 \%$ de los turistas entrevistados en este suceso oscila entre los 18 y 25 años, rango de edad que representa dependencia e independencia económica. En ese mismo rango le sigue la Disco, con el 44\%. En el siguiente rango, 26 y 35 años, donde la expectativa es que son personas con independencia económica, los porcentajes más altos que se encuentran ahí corresponden a los sucesos: Banana (68\%), ClCl (54\%), Playa (52\%) y, Hotel (51\%). Y, los mayores porcentajes de turistas cuya edad oscilaba entre los 36 y 55 años, edades en las que se supone plenitud económica, se encuentran en los sucesos: Quebrada (31\%) y Playa (30\%).

El lugar preferido para pasarla sin los familiares es en la Disco, pues el 80\% de los turistas de estudio así lo reportaron. Donde se observa que el turista se divierte más en compañía de los familiares es en: la Banana (64\%), ClCl (57\%), Quebrada (57\%) y la Playa (56\%) y, es en el Bungi (45\%) y Hotel (41\%) donde el porcentaje más alto se refiere a encontrarse en compañía de la pareja.

Llama la atención el alto porcentaje de profesionistas y estudiantes en cada uno de los siete sucesos. Así: Bungi, 100\%; Disco, 76\%; Hotel, 60\%; Playa, 58\%; Banana, 50\% y CICl, 49\%. Cabe recordar aquí que la investigación se realizó durante el período vacacional de Semana Santa.

\section{Horizonte temporal (experiencia del tiempo) de los turistas investigados}

Cada quien posee un tiempo individual (tiempo fenomenológico), por lo que cada individuo adquiere un estilo personal de interactuar y de lenguaje. Además, cada uno tiene una experiencia del tiempo, pues "el tiempo se vive". La experiencia 
acerca del tiempo no es la adquisición de una realidad externa, sino que es un constructo personal, es decir, el tiempo es generado mientras vamos construyendo la propia realidad presente (objetos y sucesos). A esa construcción de la realidad presente se le llama "presente activo", el cual es móvil y variable, pues la temporalidad de cada persona varía de acuerdo a su contexto.

Desde esta perspectiva los turistas en su interactuar y en su lenguaje reflejan fundamentalmente la experiencia de estar "libres para", no la de estar "libres de"; además, tratan de decirnos que viven el tiempo (lo experimentan) más que "viven en el tiempo". En consecuencia, sostenemos que el comportamiento general de un turista está más relacionado con el tiempo fenomenológico (tiempo del interior y presente), que con cualquier otro tipo de tiempo.

Hasta hoy en día, el tiempo de Acapulco ha sido caracterizado como el tiempo de los períodos vacacionales y de los "puentes", por lo que equivale a ser un tiempo circular o anular. Este tipo de tiempo conlleva a una disonancia temporal entre los prestadores del servicio turístico y los turistas, pues los primeros tratan de "aprovechar al máximo aquel tiempo vacacional" con fines económicos, mientras que los turistas tratan de "vivir al máximo su tiempo libre", sobre todo con la diversión. Aquí cabe recordar lo que ya se mencionó acerca de las prisas en un cierto tipo de turismo, el cual pudiera denominarse "turismo frenético", pues conduce más a insatisfacciones y al cansancio.

Entonces, de acuerdo a estas afirmaciones, veamos en la tabla 3 qué porcentaje de turistas investigados optó por la categoría "mucho" respecto a cada una de las dos coordenadas temporales (pasado-futuro) que operan en el "presente activo", teniendo en cuenta cada uno de los siete sucesos.

Tabla 3 Valoración de 'mucho' en la categoría satisfacción 


\begin{tabular}{|l|l|l|}
\hline Suceso & Futuro (\%) & Pasado (\%) \\
\hline Discoteca & 53 & 38 \\
\hline Playa & 59 & 38 \\
\hline Hotel & 56 & 43 \\
\hline ClCl & 57 & 37 \\
\hline Quebrada & 59 & 36 \\
\hline Bungi & 73 & 27 \\
\hline Banana & 64 & 32 \\
\hline
\end{tabular}

Como resultado acerca del "horizonte temporal", la tabla 3 muestra que el "presente activo" de los turistas investigados en todos los siete sucesos está impregnado más por el futuro, pues representa el $60 \%$ en promedio, en comparación con el 36\% de turistas cuya orientación fue hacia el pasado. Cabe recordar que ese porcentaje representa a la categoría "mucho". Este dato puede ser tomado en cuenta por la publicidad, sobre todo si se trata de reposicionar la imagen de un determinado lugar de Acapulco en los turistas en potencia.

En cuanto a la experiencia de cómo pasó el tiempo, si rápida o lentamente, un alto porcentaje de turistas reportó que pasó rápidamente. Así, en el Bungi, el resultado fue del 100\%, en la Disco 98\%, Hotel 97\%, la Playa 92\%, ClCl 91\%, Banana 89\% y Quebrada 88\%. Desde este punto de vista, el hecho de haber tenido la experiencia de que el tiempo pasó rápidamente, ello puede llevarnos a entender que el grado de satisfacción de las necesidades de los turistas investigados fue alto. Sin embargo, conviene tomar en cuenta los datos de las configuraciones.

\section{Resultados obtenidos a partir de cada una de las dos escalas: nominal y ordinal}


El estudio realizado acerca de la correlación entre las variables no fue para buscar la relación causa-efecto entre ellas, sino más bien, como lo afirma $\mathrm{R}$. Johnson (1996:99-101 y 463-465), para determinar el grado con el que se acompañan y la fuerza con la que lo hacen. En cuanto al cálculo de los porcentajes, tal cálculo se hizo con el fin de sintetizar y describir las frecuencias marginales en sentido relativo y no absoluto. Aparte de lo anterior, el análisis de las relaciones entre las variables también fue hecho para descubrir patrones o estructuras de relaciones.

En este apartado, el orden de la presentación tiene que ver con el tipo de escala utilizada. Por consiguiente, primero se abordarán aquellos resultados derivados de la escala nominal, tanto con variables tratadas aisladamente, como de manera conjunta y, en segundo lugar aparecerán los resultados obtenidos a través de la escala ordinal, donde las variables fueron trabajadas en forma conjunta.

\section{1.- Escala nominal}

Como en este tipo de escala importa más la posición que guarda cada una de las sub-variables, no así su orden o jerarquía, por eso mismo la siguiente presentación corresponde a la posición que cada una de aquellas tiene respecto a la autorrealización.

\subsection{1- Resultados presentados en forma aislada}

\subsubsection{1- Dimensión biológica}

Lo que más contribuyó y, con mucha fuerza, a la autorrealización de los 399 turistas, fue el haber tenido, en un determinado momento y lugar de Acapulco, la experiencia de un cuerpo "muy" saludable. 
La tabla 4 nos da una idea aproximada de cuál fue el porcentaje ${ }^{3}$ de contribución y fuerza, sólo de la categoría "mucho", en cada uno de los siete sucesos.

Tabla 4 Por ciento de valoraciones de 'mucho' en los sucesos en la Dimensión Biológica

\begin{tabular}{|l|l|l|l|l|l|l|l|l|}
\hline Indicador & Disco & Playa & Hotel & $\mathrm{CICl}$ & Quebrada & Bungi & Banana & Total \\
\hline Saludable & $\mathbf{2}$ & 65 & 67 & 62 & 66 & 43 & 79 & 66 \\
\hline Fuerte & 76 & 65 & 55 & 90 & 52 & 40 & 67 & 64 \\
\hline
\end{tabular}

De acuerdo a la tabla 4, donde menos experimentaron los turistas tener un cuerpo "muy" saludable o "muy" fuerte fue en el Bungi. Es de suponer que en este suceso predomina el estrés. Aparte del $\mathrm{CICl}$ (experiencia de un cuerpo "muy" fuerte), fue en la Disco y en la Banana donde los turistas investigados experimentaron tener un cuerpo "muy" saludable.

En conclusión, la experiencia de tener un cuerpo "muy" saludable (categoría e indicador) son las dos cosas que describen mejor lo que es la dimensión biológica. Mejor todavía, en ese mismo nivel es que la sub-variable dimensión biológica contribuye a la autorrealización de los turistas investigados.

\subsubsection{2.- Autoconcepto}

Aparte de que la experiencia de poseer un cuerpo saludable tuvo una contribución alta en la autorrealización de los turistas investigados, también la experiencia de que los demás los vieron como turistas y el verse a sí mismos como tales, contribuyó en alto grado a ella. De hecho, el indicador que lo hace

\footnotetext{
${ }^{3}$ Los porcentajes de la tabla 4 fueron calculados a partir de la muestra recogida en cada suceso particular, no de la muestra absoluta de 399. Tal cálculo, aunque se realizó de manera ponderada, no deja de tener sus riesgos, pues en cada suceso existe diferente número de entrevistados y también distinto número de casos perdidos. Sin embargo, tomados con cautela, nos dan una imagen del tipo y grado de experiencia en cada suceso.
} 
con más fuerza ( $5 \%$ versus 35\%) es el que tiene que ver con los otros (creo que los demás me ven como turista). Esto demuestra la importancia que los otros tienen sobre el yo.

Veamos en la tabla 5 con qué frecuencia respondieron los turistas de estudio, considerando cada suceso y sólo en la categoría "mucho".

Tabla 5 Porciento de valoraciones de 'mucho' en los sucesos en el autoconcepto

\begin{tabular}{|l|l|l|l|l|l|l|l|l|}
\hline Indicador & Disco & Playa & Hotel & ClCl & Quebrada & Bungi & Banana & Total \\
\hline $\begin{array}{l}\text { Me veo como } \\
\text { turista }\end{array}$ & 61 & 62 & 50 & 54 & 72 & 64 & 71 & 62 \\
\hline $\begin{array}{l}\text { Creo que me } \\
\text { ven como } \\
\text { turista }\end{array}$ & 58 & 52 & 49 & 37 & 71 & 82 & 71 & 60 \\
\hline
\end{tabular}

En la tabla 5 llama la atención que, en los sucesos: Bungi, Quebrada y Banana es donde más fueron reconocidos y tratados por los demás (no por sus familiares y amigos) como turistas; en cambio, en el $\mathrm{ClCl}$ es donde menos fueron considerados como tales. En resumidas cuentas, debido al alto grado de dependencia que hay entre los dos indicadores del autoconcepto (0.8 y 0.7 ) y por el alto grado de homogeneidad que presenta la categoría "mucho", por ello mismo se puede sostener que el autoconcepto de los turistas estudiados contribuye en un nivel alto en su autorrealización, sobre todo a partir de que los demás así los vieron.

Estos resultados sugieren la necesidad de profesionalizar I trato a los turistas

\subsubsection{3.- La Plenitud.}

Este indicador ocupa el tercer lugar en importancia en la valoración de los encuestados. Los turistas de este estudio experimentaron, en grado REDMARKA UIMA-Universidad de A Coruña - CIECID 93 Año 1, Número 2, (2009), pp. 77-110 
relativamente alto (0.69 y 0.66), que su vida estaba llena de significados. Entonces, en ese mismo grado esta sub-variable contribuyó a su autorrealización. A decir verdad, la posición relativamente alta es poco significativa, ya que su vida está llena de significados es un ingrediente importante de la autorrealización.

Tomando en cuenta que en la sub-variable plenitud las categorías "mucho" y "regular", ambas suman casi el 100\%, veamos en la tabla 6, desde el cálculo de frecuencias marginales, en qué porcentaje se dieron esas categorías en cada uno de los siete sucesos. Los porcentajes del lado izquierdo representan la categoría "mucho", los de la derecha a la categoría "regular"

Tabla 6 Porciento de valoraciones de 'mucho' en los sucesos para la plenitud

\begin{tabular}{|c|c|c|c|c|c|c|c|c|}
\hline Indicador & $\begin{array}{l}\text { Disco } \\
\%\end{array}$ & Playa & Hotel & $\mathrm{ClCl}$ & Quebrada & Bungi & Banana & Promedio \\
\hline Significados & $\begin{array}{l}60- \\
33=93\end{array}$ & $\begin{array}{l}48- \\
34=82\end{array}$ & $\begin{array}{l}43- \\
40=83\end{array}$ & $\begin{array}{l}46- \\
34=80\end{array}$ & $64-31=95$ & $\begin{array}{l}64- \\
36=100\end{array}$ & $\begin{array}{l}75- \\
21=96\end{array}$ & $57-33$ \\
\hline Sentimientos & $\begin{array}{l}71- \\
18=89\end{array}$ & $\begin{array}{l}65- \\
22=87\end{array}$ & $\begin{array}{l}49- \\
34=83\end{array}$ & $\begin{array}{l}54- \\
31=85\end{array}$ & $62-24=86$ & $\begin{array}{l}55- \\
45=100\end{array}$ & $\begin{array}{l}67- \\
26=93\end{array}$ & $60-29$ \\
\hline
\end{tabular}

En realidad, los promedios de la tabla 6 confirman el valor medianamente alto de la experiencia de plenitud. En cuanto a la categoría "mucho", en cada suceso tenemos que, es en la Banana donde los turistas investigados presentaron, en un porcentaje alto (75\%), que su vida, "mucho" estaba llena de significados. También en grado alto (71\% "mucho") se observa que en la Disco es donde los turistas afirmaron que aceptaban sus sentimientos tal y como eran, sin asustarse de ellos, aunque no fuesen socialmente aceptados. Los porcentajes encerrados en un círculo representan el nivel más bajo de la categoría "mucho". Entonces, en el Hotel, el $\underline{\mathrm{CICl}}$ y la Playa es donde pocos de los turistas de estudio experimentaron que "mucho" su vida estaba llena de significados. En otras palabras, ahí pocos turistas lograron "mucha" autorrealización. 


\subsubsection{4.- La Autodeterminación}

Este indicador fue ubicado en cuarto lugar en la encuesta. La experiencia de los turistas investigados acerca de su tiempo libre, contribuyó en forma regular en su autorrealización. Además, en la conformación de esa experiencia del tiempo libre, el que más participó y con más fuerza fue el indicador lo hace libremente, con el 46\%, mientras que el indicador diversión lo hizo con el 25\%. Entonces, de acuerdo a esto se puede afirmar que, el tiempo libre del turista es la experiencia del tiempo para: poner las condiciones de un cuerpo saludable, para redefinirse a sí mismo, (en este caso como turista), para llenar su vida de significados y para la diversión.

Esto quiere decir que, el tiempo y la libertad para de tales turistas, están ahí, en gran medida, como unas de las condiciones para ser turista $y$, en grado moderado, como aquello que conduce a una experiencia de autorrealización (vivir el tiempo).

Ahora bien, ¿En qué grado lograron esa experiencia de libertad para la diversión? Supuesto que en la sub-variable autodeterminación la categoría "mucho", comparada con "regular" y "poco" es la más alta, por eso mismo la tabla 7 muestra en porcentajes cómo fue la preferencia de los turistas de estudio respecto a la tres "D", en cada uno de los siete sucesos. Los porcentajes altos aparecen encerrados en un óvalo y en un rectángulo.

Tabla 7 Por ciento de valoraciones de 'mucho' en los sucesos en autosatisfacían

\begin{tabular}{|l|l|l|l|l|l|l|l|l|}
\hline Indicador & Disco & Playa & Hotel & $\mathrm{ClCl}$ & Quebrada & Bungi & Banana & Promedio \\
\hline Descanso & 61 & 56 & 67 & 62 & 62 & 50 & 67 & $61 \%$ \\
\hline Diversión & 91 & 79 & 81 & 68 & 75 & 89 & 92 & $82 \%$ \\
\hline $\begin{array}{l}\text { Desarrollo } \\
\text { personal }\end{array}$ & 70 & 39 & 41 & 59 & 43 & 25 & 35 & $45 \%$ \\
\hline
\end{tabular}




\begin{tabular}{|l|l|l|l|l|l|l|l|l|}
\hline $\begin{array}{l}\text { Lo hace } \\
\text { libremente }\end{array}$ & 77 & 64 & 66 & 57 & 79 & 82 & 75 & $71 \%$ \\
\hline
\end{tabular}

Por los datos de la tabla 7 se puede afirmar que, los turistas investigados, donde "mucho" se divirtieron fue en la Banana, la Disco y en el Bungi. Por otro lado, considerando los promedios de la categoría "mucho" en esta misma tabla, puede observarse que es la diversión la que más peso tiene. Esto quiere decir que tales turistas pueden llegar a Acapulco cargados de aburrimiento, al cual le hacen frente mediante una experiencia de "mucha" diversión. Es notorio cómo en el Bungi nada que ver el desarrollo personal (25\%). Cabe recordar que, aunque los porcentajes de la diversión son altos, ello no significa que en esa medida contribuyeron a la autorrealización, pues como ya se dijo anteriormente, la aportación de la autodeterminación para la autorrealización fue regular.

\subsubsection{5.- La Satisfacción de la Necesidad}

Esta sub-variable ocupa la posición más distante, por lo que su contribución a la autorrealización de los turistas investigados fue ligeramente baja. En realidad, al igual que en el autoconcepto y en la plenitud, en la satisfacción de la necesidad las categorías "mucho" y "regular", que por cierto son las que representan el mayor porcentaje, "jalan parejo", es decir, se reparten las frecuencias. Este resultado, a primera vista, no es nada halagüeño, sobre todo si consideramos que la satisfacción es una parte fundamental de la autorrealización. Sin embargo, cuando se mide el grado de satisfacción de alguna necesidad, es necesario tener en cuenta ciertos factores, algunos de los cuales incluso pareciera que no tuviesen que ver con ella. Por ejemplo, el lugar, el momento, las fantasías, tipos de necesidades, ${ }^{4}$ el nivel mismo de la satisfacción, entre otros. Ahora bien, supuesto que esta parte de los resultados que hemos venido analizando tiene como base la escala nominal, por ello mismo importa más

\footnotetext{
${ }^{4}$ En este trabajo son las necesidades diseñadas por A. Maslow las que nos sirven como marco de referencia (Cfr. figura 1: Jerarquía teórica de las necesidades).

REDMARKA UIMA-Universidad de A Coruña - CIECID Año 1, Número 2, (2009), pp. 77-110 
determinar cuál es la posición que guardan algunos de esos factores respecto a la satisfacción y, en consecuencia, en relación con la autorrealización de los turistas. En consecuencia, ya que la satisfacción de las necesidades propias de un turista fue lograda en un grado ligeramente bajo, por ello mismo la autorrealización presentará también una tendencia hacia el nivel "regular".

\subsubsection{Resultados conjuntos}

De estos resultados presentados en forma aislada se puede afirmar lo siguiente:

a) No es muy alentador el resultado de que la satisfacción de las necesidades (en promedio equivale al 13.5\%) de los turistas investigados en determinados lugares de Acapulco haya sido ligeramente baja. Desde la perspectiva fenomenológica esto equivale a decir que, la congruencia entre el yo real de los turistas y su yo ideal también fue ligeramente baja. Puede resultar interesante investigar si ese $13.5 \%$ es precisamente el porcentaje de turistas que regresan a Acapulco. De ser así, entonces tenemos un turismo reciclado, lo cual pudiera producir cierto estancamiento en la actividad turística.

b) Los siete lugares de Acapulco analizados en este estudio, cumplen una función importante en la satisfacción de las necesidades propias del turista. Los dos indicadores de esta sub-variable que se refieren al lugar participan con el $40 \%$ en la satisfacción de la necesidad (26\% y $14 \%$ respectivamente), porcentaje relativamente alto.

c) Los "sueños" o fantasías (expectativas) que los turistas esperaban ver realizados cuando se encontraran en un determinado momento y lugar de Acapulco fueron alcanzados en un nivel bajo, 16\%. Ahora, dentro de este $16 \% \mathrm{y}$, teniendo en cuenta la categoría "mucho", veamos en qué grado lograron sus fantasías en cada uno de los siete sucesos. Donde más las alcanzaron fue en el Bungi, con un 73\%, seguido de la Banana con el $61 \%$, el $\mathrm{ClCl}$ con el 51\%, la Playa y en la Quebrada, ambas con el 45\%, 
la Disco con el $44 \%$ y en el Hotel con el $43 \%$. Ojala un estudio posterior nos explique qué es lo que puede estar sucediendo respecto a ese nivel bajo de satisfacción.

d) Nos queda claro que los turistas investigados en el Acapulco Dorado y Tradicional (Náutico), durante el período vacacional de Semana Santa, no vienen con el fin de ostentar un rango social (estatus social). Del 7\% de esta necesidad, en el Bungi es donde más se presentó, ya que alcanzó el $64 \%$ dentro de la categoría "mucho" y, donde menos se expresó fue en El Hotel, ahí su nivel fue del $17 \%$.

En general, las cinco variables analizadas anteriormente contribuyen a la autorrealización en un nivel moderado (.6 y .5).

Considerando que la posición de una variable afecta la posición del resto de las variables, veamos qué resultados encontramos en cuanto a la contribución de cada una de ellas en relación a la autorrealización, pero considerándolas ahora de manera conjunta. Desde esta otra perspectiva las posiciones obtenidas son: El autoconcepto ocupa aquí la primera posición, por lo que es la variable que más aporta a la autorrealización de los turistas investigados, haciéndolo con el $34 \%$; le sigue la sub-variable plenitud, con el 28\%, en tercer lugar la satisfacción de la necesidad con 17\%, luego la dimensión biológica con el 12\% y finalmente la autodeterminación con el 9\%

Definitivamente es el hecho de verse a sí mismos como turistas (el autoconcepto) lo que determina más la autorrealización de los turistas investigados. Pero además, son precisamente los otros, no tanto el yo, los que le dan fuerza a esa autodefinición. En otras palabras, en la autorrealización de tales turistas contó en buena medida el que los otros los hayan tratado precisamente como turistas. 
En el segundo plano de contribución a la autorrealización se encuentra la experiencia de haber tenido en ese momento y en ese lugar una vida llena de significados, una vida llena de estímulos personales. Si aunamos a esto, que el lugar jugó un papel importante en la autorrealización de los turistas entrevistados, se puede afirmar entonces que estos mismos turistas se entregaron a lo que les rodeaba, expandieron y enriquecieron sus experiencias, incrementando así su libertad.

Otra cosa que está presente en esa visión de conjunto es la experiencia que tuvieron acerca de su tiempo como tiempo libre. Resulta ser que este tipo de tiempo no fue tan importante para su autorrealización, pues ocupó la última posición. En cuanto a la posición que ocupan, tanto la satisfacción de la necesidad como la dimensión biológica, no es muy clara como lo fue la de las sub-variables anteriores; no obstante, la satisfacción de la necesidad puede quedar ubicada en la tercera posición.

\section{2.- Escala ordinal}

El resultado más importante de esta escala es el de la autorrealización en forma general, es decir, la que deriva de la relación de las sub-variables entre sí y, de éstas con el autoconcepto. De hecho, es lo que hemos venido esperando encontrar.

Tabla $8^{a}$ Resultados de los ordenación de las variables

\begin{tabular}{|c|c|c|c|c|c|c|c|c|}
\hline \multirow{2}{*}{ Variable } & \multirow{2}{*}{ Mínimo } & \multirow{2}{*}{ Máximo } & \multirow{2}{*}{ Media } & \multirow{2}{*}{ Error típico } & \multirow{2}{*}{ Desv. típ. } & \multicolumn{2}{|c|}{ Intervalo de confianza } & \multirow{2}{*}{ Calificación } \\
\hline & & & & & & $\mathrm{LI}$ & LS & \\
\hline Puntuación dimención biológica & 2 & 8 & 5.43 & 0.11 & 2.03 & 5.21 & 5.64 & 6.78 \\
\hline Puntuación Autodeterminación & 4 & 16 & 10.77 & 0.17 & 3.48 & 10.42 & 11.12 & 6.73 \\
\hline Puntuación Autorealización & 4 & 8 & 6.80 & 0.07 & 1.39 & 6.66 & 6.94 & 8.50 \\
\hline Puntuación Autoconcepto & 4 & 8 & 6.74 & 0.08 & 1.59 & 6.58 & 6.90 & 8.42 \\
\hline Puntuación satisfacción & 6 & 24 & 20.28 & 0.14 & 2.79 & 20.00 & 20.56 & 8.45 \\
\hline Puntuación total & 16 & 64 & 49.61 & 0.38 & 7.59 & 48.85 & 50.37 & 7.75 \\
\hline
\end{tabular}


La tabla $8 b$, cuyos datos provienen de la tabla $8 a$, presenta tres columnas básicas: la $\delta$ es la desviación típica, es decir, qué tanto se desvía de la norma, que en este caso es la autorrealización general; la calificación representa la media de cada variable convertida en una escala del 0 al 10 y, la columna de las categorías, esta última para darnos cuenta de qué porcentaje de turistas optó por los valores "muy" y "regular".

Tabla 8b Resultados estadísticos de los ordenación de las variables

\begin{tabular}{|c|c|c|c|c|c|}
\hline Variable & $\delta$ & Calificación & $\begin{array}{l}\text { Categ } \\
=\text { Total }\end{array}$ & & aceptable \\
\hline $\begin{array}{l}\text { Autorrealización en } \\
\text { general }\end{array}$ & 7.59 & 7.75 & $17 \%$ & $36 \%$ & $53 \%$ \\
\hline Autodeterminación & & & $22 \%$ & $28 \%$ & $50 \%$ \\
\hline Plenitud & 1.39 & 8.5 & 0 & $66 \%$ & $66 \%$ \\
\hline $\begin{array}{l}\text { Satisfacción de la } \\
\text { necesidad }\end{array}$ & 2.79 & 8.45 & $11 \%$ & $41 \%$ & 52 \\
\hline Dimensión biológica & & & $28 \%$ & $16 \%$ & $44 \%$ \\
\hline Autoconcepto & 1.59 & 8.42 & 0 & $67 \%$ & $67 \%$ \\
\hline
\end{tabular}

Por lo que se puede observar en la tabla 8b, el nivel de autorrealización alcanzado por los turistas investigados es regular, ya que la categoría que prevalece es la de aceptable, que sumada a la "mucho" da el total de 53\%. Sin embargo, su calificación es casi de 8. Estos dos resultados no se oponen mutuamente, más bien son complementarios.

Respecto a las variables que más contribuyeron al nivel alcanzado de la autorrealización, vuelven a ser las mismas que ya anteriormente se han venido mencionando, esto es, la plenitud y el autoconcepto. La satisfacción de la necesidad, aunque su calificación es alta (8.45), no obstante su categoría 
"regular" (que es la más alta de las dos) es ligeramente baja (41\%), por lo que su tendencia es hacia el nivel bajo.

Fue en la Banana y en la Disco donde al nivel de "mucho" se sintieron autorrealizados los 399 turistas (36\% en cada suceso), siguiéndolos el $\mathrm{CICl}$ con el 26\%. En la Playa, sólo el $14 \%$ de ellos experimentaron "mucha" autorrealización y en el Bungi ninguno la experimentó en ese grado.

Supuesto que el nivel de autorrealización fue regular, si se suma esta última categoría a de "mucho" tenemos que: es en la Banana donde los turistas alcanzaron el mayor nivel de autorrealización, 82\%; le sigue la Disco con el 69\%. Los lugares donde menos la experimentaron fueron: Bungi con 45\%, Quebrada con el $42 \%$ y el Hotel con el $41 \%$.

5.3 Hechos importantes a tomar en cuenta en la proyección de la mejoría de la imagen

El hecho de que la autorrealización haya sido alcanzada por los turistas investigados en un nivel regular (53\%) no es nada satisfactorio. Si se vuelve a revisar el gráfico 13 se podrá observar que los cinco conjuntos caen dentro de un rango comprendido entre el $44 \%$ y el $67 \%$, porcentajes equivalentes a los valores 0.6 y 0.5 .

En realidad, ese nivel regular va paralelo al grado de satisfacción de las necesidades como turistas, ya que sólo el 11\% de ellos reportaron que "mucho" la alcanzaron. Realmente ese porcentaje no es significativo, por el contrario, es demasiado poco.

Los lugares donde los turistas de estudio experimentaron mayor grado de autorrealización (aunque en ese nivel regular) son: 
1.- Banana con $82 \%$

2.- Disco, $69 \%$

3.- $\mathrm{ClCl}, 55 \%$

4.- Playa, $53 \%$

5.- Bungi, $45 \%$

6.- Hotel y Quebrada, ambos con $41 \%$

En la tabla 10, cada uno de los siete sucesos aparece relacionado con aquellos indicadores que presentaron mayor porcentaje (arriba de 70\%) dentro de la categoría "mucho".

Tabla 10 Indicadores con alto porcentaje de valoraciones de 'mucho'

\begin{tabular}{|l|l|}
\hline Disco & * Saludable, sentimientos, diversión, libremente \\
\hline Playa & Diversión \\
\hline Hotel & Diversión \\
\hline $\mathrm{ClCl}$ & Fuerte \\
\hline Bungi & Diversión, libremente, me ven, fantasías. \\
\hline Quebrada & Diversión, libremente, me ven, me veo \\
\hline Banana & $\begin{array}{l}\text { Saludable, diversión, libremente, me ven, me veo, fantasías, } \\
\text { significados }\end{array}$ \\
\hline
\end{tabular}

* Conceptos que describen algunos de los siguientes indicadores que aparecen en la tabla 10:

-Saludable: El turista experimentó que en ese lugar su cuerpo era saludable y/o fuerte.

-Sentimientos: Al experimentarlos en ese lugar los aceptaba, independientemente de cuáles fueran.

-Libremente: Experimentó libertad para...

-Me veo, me ven: En ese lugar se percibía a sí mismo como turista y/o percibió que los demás lo veían como tal. 
-Fantasías: Si es que fueron cumplidas o no estando en ese lugar.

-Significados: En ese lugar experimentó que su vida estaba llena de significados

En consecuencia, es en la Banana donde los turistas investigados experimentaron mayor amplitud de autorrealización y, qué lástima que en la Playa haya habido cierto nivel de pobreza en cuanto a experiencias.

Respecto a los factores que más influyeron en la autorrealización de los turistas investigados, no cabe duda de que el percibirse a sí mismos como turistas (autoconcepto) eso fue lo más determinante, considerando un poco más aquella percepción que proviene de los otros, no tanto del yo. En otras palabras, si el visitante percibe que, los que le prestan un servicio directamente, se lo ofrecen precisamente como turista (y lo que ello implica), eso es un factor importante para su autorrealización.

Casi igual que el autoconcepto, la experiencia de que su vida estaba llena de significados (plenitud) también contribuyó con valores altos a la autorrealización de los 399 turistas. Parafraseando a Maslow (2006:57-58), el turista es una persona en proceso de autorrealización, que se consagra y se sumerge completamente en una experiencia plena, vívida y personal del momento y del lugar, fuera de los excesos del autocontrol y de la autoconciencia. En ese momento de experiencia, el turista es una persona total y plenamente humana.

Aparte de la imagen que tuvieron acerca de sí mismos como turistas y el haber tenido experiencias de que su vida estaba llena de significados, el hecho de haber experimentado en ese momento y en ese lugar que su cuerpo era saludable, también esto último contribuyó de manera importante a su autorrealización. De ahí que puede afirmarse que, quienes visitan Acapulco como turistas son personas físicamente sanas. Sobre esta afirmación, puede 
hacerse otra afirmación más: aparte del tiempo libre y del factor económico, otro determinante para ser turista es que se experimente físicamente sano.

¿Y qué decir de la satisfacción de las necesidades propias de un turista? El nivel alcanzado por los turistas de estudio en esta sub-variable deja mucho qué pensar (52\%, lo que equivale a un nivel regular). A este respecto conviene hacer algunas precisiones acerca de sus indicadores.

Queda claro que los turistas que visitaron los siete lugares de Acapulco, estudiados en esta tesis, poco buscaron y/o encontraron la satisfacción de su necesidad de estima. ${ }^{5}$ De hecho, de los seis indicadores que forman la subvariable satisfacción, precisamente los dos que están relacionados con la aceptación y el reconocimiento por parte de los demás, son los que menos contribuyen a la satisfacción de los turistas.

Por los datos analizados hasta aquí, estamos seguros de que pocos (no sus familiares y/o amigos) prestaron atención a dos cualidades que el turista esperaba se les tomasen en cuenta y por las cuales experimentaría ser estimado y apreciado. Nos referimos a la temporalidad y libertad del turista. En general, todo turista es una persona que vive el tiempo, además de poseer libertad para. Entonces, como tales cualidades poco fueron atendidas, por eso mismo la satisfacción de la necesidad de estima y ser reconocido poco llegó a su esfera de la experiencia.

Ahora bien, como una consecuencia lógica del bajo nivel de satisfacción de la necesidad de estima, resulta que, el cumplimiento de sus fantasías (lo que esperaban encontrar en ese momento y en ese lugar) también haya sido ligeramente bajo.

\footnotetext{
${ }^{5}$ Es una de las necesidades propias del turista y tiene que ver con la necesidad de ser reconocido por algo que se tiene, ya sea una cualidad personal, ya su situación económico-social o, lo que esta tesis considera lo más importante del turista, su tiempo libre para.

REDMARKA UIMA-Universidad de A Coruña - CIECID Año 1, Número 2, (2009), pp. 77-110

104 http://www.redmarka.org/
} ISSN 1852-2300 
Entonces, el tiempo del turista es una cuestión sumamente importante, pues creemos que, en la medida que haya una sincronía entre los dos tiempos, el tiempo del turista y el tiempo de los prestadores del servicio turístico, en esa misma medida el turista avanzará más veces hacia su autorrealización, hacia su expansión; encontrará así mayor libertad para escuchar la voz de su propio yo y no la voz de mamá o de quién sea. Es decir, teniendo el turista sincronía en los dos tiempos, entonces verá en Acapulco lo que él quiere ver de él, no lo que le dicen que vea (por ejemplo la publicidad), experimentará los sentimientos que él quiere sentir en Acapulco (no importa cuáles sean), no los que le dicen que sienta. Este es el verdadero sentido de la tesis, o sea, aquí no se trata de un tiempo libre traducido simplemente como un tiempo fuera del trabajo o libre de las obligaciones, no es una simple libertad de, sino la trascendencia y la expansión del yo. Por eso es que temporalidad y libertad van de la mano. Estamos seguros de que si los turistas experimentan una sincronía de esos dos tiempos, ello contribuirá en buena medida en la solución de algunos problemas relacionados con la actividad turística.

En cuanto al papel que juega el lugar en la satisfacción de los turistas tenemos que, son precisamente los indicadores que se refieren a él los que presentan el más alto grado de contribución. En consecuencia, el nivel regular de satisfacción alcanzado por los turistas de estudio no se debió a un "desencanto" por haber encontrado un lugar distinto al esperado, sino más bien porque esperaban ser atendidos como personas que están en proceso de autorrealización.

Resulta ser bastante elocuente y hasta paradójico, cómo la autodeterminación (elemento que soporta al concepto tiempo libre), siendo parte fundamental en el estudio y comprensión del TURISMO, es de las su-variables que ocupan las posiciones más alejadas, por lo que es la que menos aporta a la autorrealización de los turistas investigados. Como se acaba de mencionar anteriormente, dicha posición alejada sólo puede ser entendida desde el hecho de que tales turistas

REDMARKA UIMA-Universidad de A Coruña - CIECID 105 Año 1, Número 2, (2009), pp. 77-110 http://www.redmarka.org/ ISSN 1852-2300 
poco fueron atendidos en su temporalidad (la cual es individual y personal) y en su libertad para.

En resumidas cuentas, los 399 turistas investigados en siete lugares representativos de Acapulco, lograron en un nivel moderado su autorrealización. En consecuencia, la congruencia entre su yo real y su yo ideal se quedó apenas un poco arriba de la mitad.

\section{Conclusiones del estudio}

Ante el resultado de que la autorrealización de los 399 turistas fue lograda en un nivel regular, surgen algunas interrogantes. Por ejemplo: ¿Será que, en la mayoría de los turistas que visitan Acapulco predomine la superficialidad en vez de que su estancia les represente una oportunidad para experimentar el desarrollo de ciertas capacidades? ¿Acaso lo que más les interese sea un máximo de bienestar físico (cuerpo saludable) en lugar de convertirse en personas totales y plenamente humanas, capaces de "abandonarse" (sumergirse) a sus experiencias del momento? Estas y otras preguntas parecidas emergen a raíz del nivel regular en el que fue alcanzada la autorrealización.

En cuanto al logro ligeramente bajo de sus fantasías, (poco se cumplió de aquello que esperaban encontrar), es posible que algún turista se haya propuesto metas utópicas (inalcanzables), lo que evidentemente conduce a una gran insatisfacción, no por lo que no encontró, sino por lo inalcanzable de su meta. Sin caer en los extremos y porque no hubo un control real de ello se puede asegurar que eso, poco o nada sucedió, pues la pregunta seis del cuestionario involucra más al lugar, no a un plan de vida. Toca a elaborar ofertas que den satisfacción a otros anhelos a los que se ha preparado el mercado turístico de este polo. 
Cabe hacer mención del papel importante que juega el lugar en las experiencias del turista. En este punto queremos hacer una reflexión. Los siete sucesos, como lugares mismos ahí están, ellos tienen su propio valor y significado. Con riesgo de caer en un equívoco, consideramos que para los turistas en general, la contaminación (basura en la playa, ruido y humo en la disco, entre otras cosas), independientemente del grado de toxicidad, no es lo primero que entra a la esfera de su experiencia, sino el lugar en su conjunto. Evidentemente que esto no es una justificación del grado de contaminación que halla en Acapulco, es más bien el impacto que tiene el lugar en el campo de la experiencia, cuando el turista se encuentra en Acapulco. Una labor más fuerte es necesaria de todos modos en el saneamiento de las playas.

Finalmente, por el tipo de variables que hemos manejado y por los resultados obtenidos, este trabajo puede coadyuvar en algunas áreas de la actividad turística, como son: la capacitación de los prestadores del servicio, el diseño de una publicidad que incida sobre la temporalidad y la libertad de turistas en potencia y, apoyando los proyectos que la Dirección General de Turismo Municipal emprenda y que estén relacionados con un trato diferente hacia el turista.

\section{Recomendaciones para elaborar una política mercadeo}

Hay una frase que comúnmente se escucha en Acapulco: "Hay que tratar bien al turista". La frase en sí es cierta, pero es tan general, que se descalifica por sí misma. Existen varios hechos que demuestran el buen trato que se ofrece a los turistas. Sin embargo, como resultado de todo este trabajo se puede recomendar que, en lugar de ofrecer el clásico "buen trato" es más útil tratar al turista precisamente como turista. Esa es la clave, pues resulta que sólo el $9 \%$ de ellos manifestó ser reconocido y aceptado como tal, por parte de los demás, excepto sus familiares y amigos. Tratarlos como turistas implica:

REDMARKA UIMA-Universidad de A Coruña - CIECID Año 1, Número 2, (2009), pp. 77-110 107 http://www.redmarka.org/ ISSN 1852-2300 
a) Reconocer su temporalidad (tiempo fenomenológico, tiempo de su realidad interior), por lo que hace necesaria la sincronización.

b) Aceptar su libertad para, lo cual es traducido como creatividad; libertad para expresar sus sentimientos, sin fingir o adoptar poses, sino conociendo el momento de la responsabilidad; libertad para significa atreverse a escucharse a sí mismo, escuchar su propio gusto, no lo que la publicidad dice que debe gustar, abrirse a uno mismo.

c) Redescubrir los significados y las expectativas que tienen acerca de los lugares más representativos de Acapulco.

En resumidas cuentas, se sugiere un nuevo trato, donde se tomen en cuenta las cualidades por las que debe ser estimado y reconocido. Ciertamente hay ejemplos loables del buen trato hacia los turistas. De hecho, hay empresas turísticas cuyos servicios son de calidad, ya que tratan al turista como una persona "distinguida", por mencionar algo. Desde esta tesis, ese estilo de trato no basta, es necesario tratarlo precisamente como turista en Acapulco.

Finalmente, el TURISMO, por un lado, es una de las oportunidades que las personas tenemos para vivir cada momento, cada circunstancia, cada lugar, no porque tengamos tiempo libre de, por ejemplo, de los automatismos del trabajo, sino sobre todo porque el tiempo es existencia, el tiempo se vive; por otra parte, el turismo es uno de tantos momentos de la vida en el que se experimenta libertad para, principalmente para la diversión, el descanso y para el desarrollo humano.

\section{BIBLIOGRAFÍA}

Cook, Ch. S. (2000) Métodos cualitativos y cuantitativos en investigación educativa. Ed. Morata, $3^{\mathrm{a}}$ reimpresión, Madrid. 
Ferran A. M. (2001). SPSS. Análisis estadístico para Windows. Ed. Mc. Graw Hill.

Frager, R. y Fadiman, J. (2007) Teorías de la Personalidad. Segunda edición, séptima reimpresión, Oxford, University Press \& Alfaomega, Grupo Editor. Julio, México.

Guerrero Ocampo, M. (2008) La Esfera De La Experiencia Del Turista En Acapulco

Estudio Fenomenológico. Tesis de Doctorado, Universidad Autónoma de Guerrero.

Johnson, R. (1996). Estadística Elemental, Grupo Editorial Iberoamérica, México.

Kotler, .P. "Dirección de Marketing. La edición del milenio" Ed. PrenticeHall, México., 2001.

Levy, P. S. y S. Lemeshow (2008): Sampling of Populations: Methods and Applications, Solutions Manual, 4th Edition. Wiley, Chichester

Munné F. (2004). Psicosociología del tiempo libre. Un enfoque crítico. Undécima reimpresión, Trillas.

Rimm, D. (1981), Terapia de la conducta -Técnicas y hallazgos empíricos-. Trillas

Rivas, Teresa (1997). Tesis: Factores que influyen en el retorno del turista internacional a Acapulco.

Rueda García, M. del M. y A. Arcos Cebrián (1998): Problemas de muestreo en poblaciones finitas Grupo Editorial Universitario, Granada.

Visauta, V. . (2005). Análisis estadístico con SPSS para Windows. Segunda edición, McGraw-Hill, Méx.

Wayne W. D. (2002). Bioestadística -Base para el análisis de las ciencias de la salud-. Limusa.

Weiers, R. M. (2000). Investigación de mercados. Prentice-Hall Hispanoamericana, Méx.

Zanutto, .L.y E.T. Bradlow (2006): Data pruning in consumer choice models. Quantitative Marketing and Economics, 4, 267-287 


\section{Para citar este artículo:}

Guerrero Ocampo, Miguel - Moreno, Agustín Santiago - Bouza Herrera, Carlos . EL MERCADO TURISTICO DE ACAPULCO: ESTUDIO DEL PERFIL DE LOS TURISTAS.

REDMARKA - CIECID - Unidad de Investigación en Marketing Aplicado-Universidad de A Coruña, Número 2, V1, pp.77-110

ISSN 1852-2300

URL del Documento : http://www.cienciared.com.ar/ra/doc.php?n=1097 